\title{
Article \\ A Repeatable and Reproducible Digital Method to Quantify the Cement Excess and Enamel Loss after Debonding Lingual Multibracket Appliance Therapy
}

\author{
Alba Belanche Monterde ${ }^{1}$, Alberto Albaladejo Martínez ${ }^{1}$, Alfonso Alvarado Lorenzo ${ }^{1}$, Adrián Curto ${ }^{2}$, \\ Jorge Alonso Pérez-Barquero ${ }^{3}$, Clara Guinot-Barona ${ }^{4, *}$ and Álvaro Zubizarreta-Macho ${ }^{5}$ \\ 1 Department of Orthodontics, Faculty of Medicine and Dentistry, University of Salamanca, \\ 37008 Salamanca, Spain; belanche.alba@usal.es (A.B.M.); albertoalbaladejo@hotmail.com (A.A.M.); \\ alfonsoalvaradolorenzo@gmail.com (A.A.L.) \\ 2 Department of Surgery, Faculty of Medicine and Dentistry, University of Salamanca, 37008 Salamanca, Spain \\ adrian_odonto@usal.es \\ 3 Department of Stomatology, Faculty of Medicine and Dentistry, University of Valencia, 46010 Valencia, Spain; \\ jorgealonso86@gmail.com \\ 4 Department of Orthodontics, Faculty of Medicine and Health Sciences, Catholic University of Valencia, \\ 46001 Valencia, Spain \\ 5 Department of Endodontics, Faculty of Health Sciences, Alfonso X El Sabio University, 28691 Madrid, Spain; \\ amacho@uax.es \\ * Correspondence: clara.guinot@ucv.es; Tel.: +34-963637412
}

check for

updates

Citation: Belanche Monterde, A.; Albaladejo Martínez, A.; Alvarado Lorenzo, A.; Curto, A.; Alonso Pérez-Barquero, J.; Guinot-Barona, C.; Zubizarreta-Macho, Á A Repeatable and Reproducible Digital Method to Quantify the Cement Excess and Enamel Loss after Debonding Lingual Multibracket Appliance Therapy. Appl. Sci. 2021, 11, 1295. https:// doi.org/10.3390/app11031295

Academic Editor: Paola Gandini

Received: 12 January 2021

Accepted: 26 January 2021

Published: 1 February 2021

Publisher's Note: MDPI stays neutral with regard to jurisdictional claims in published maps and institutional affiliations.

Copyright: (c) 2021 by the authors Licensee MDPI, Basel, Switzerland. This article is an open access article distributed under the terms and conditions of the Creative Commons Attribution (CC BY) license (https:/ / creativecommons.org/licenses/by/ $4.0 /)$.

\begin{abstract}
The aim of the present study is to present a repeatable, reproductible, and accurate morphometric measurement method for measuring and quantifying the area and volume of cement that remains after fixed lingual multibracket appliance debonding, enamel loss after fixed lingual multibracket appliance debonding, and the volume of cement used to adhere fixed lingual multibracket appliances. Ten conventional lingual brackets were cemented in 10 extracted teeth embedded into an epoxy resin model simulating a dental arch. This model was scanned before and after bonding the lingual brackets, after debonding, and after polishing the surfaces. We also performed a Micro-Computed Tomography scan of the lingual brackets used. Afterward, the standard tessellation language (STL) digital file was aligned, each tooth was segmented individually, and the file was re-aligned using engineer morphometry software. Inter-operator and intra-operator comparative analyses were performed using the ANOVA test, and the repeatability and reproducibility of the morphometric measurement technique were analyzed using Gage R\&R statistical analysis. Repeatability showed $0.07 \%$ and $0.16 \%$ variability associated with the area and volume measures, respectively, while reproducibility showed $0.00 \%$ variability associated with the area and volume measures, respectively. In conclusion, the morphometric measurement technique is a repeatable, reproductible, and accurate morphometric measurement method for quantifying the area and volume of cement that remains after fixed lingual multibracket appliance debonding, enamel loss after fixed lingual multibracket appliance debonding, and the volume of cement used to adhere fixed lingual multibracket appliances.
\end{abstract}

Keywords: lingual orthodontics; debonding; cement; technique; morphometry

\section{Introduction}

Lingual orthodontics has emerged as an aesthetic system for performing multibracket appliance therapy and has been widely accepted by patients of all ages [1,2]. This technique also represents an alternative to vestibular multibracket appliances in cases with a high risk of developing white spots, allowing one to avoid this unaesthetic effect at the end of the orthodontic treatment [3]. However, lingual multibracket appliance therapy suffers from worse accessibility and visibility than conventional buccal multibracket 
appliance therapy. Furthermore, the irregular anatomy of the lingual surfaces of the upper and lower incisors may make this therapy difficult [4]. Achieving good adhesion on lingual surfaces does not seem to depend on the adhesive material used [5]. Brosh et al. reported significantly higher debonding strength and fewer bonding remnants in buccal than lingual multibracket appliances. There is, moreover, less dissolution of enamel prisms on the lingual surfaces in comparison with buccal surfaces after acid etching [6]. Therefore, sandblasting is commonly used to achieve better retention. However, this method's efficacy is controversial since Lopes et al. did not show a significant increase in shear bond strength after sandblasting pre-conditioning [7] when compared to Lombardo et al., who reported an increase in micro-retention [8]. On the other hand, lingual multibracket appliance therapy debonding produces enamel damage, which varies depending on the surface [6]. Eichenberger et al. reported a roughness increase $(\Delta \mathrm{Sa})$ of the enamel surface after lingual multibracket appliance therapy debonding of $17.16 \mathrm{~nm}$ [9]. Composite and fiber glass burs have been proposed as ways to remove the cement remaining after lingual multibracket appliance therapy debonding, as both have shown lower values of enamel roughness [9] compared to carbide-tungsten burs [10,11]. The application of solvent agents to remove adhesive remains has also been reported, and an adhesive residue remover has been recommended because it causes less damage to the enamel surfaces [11] However, the polishing procedures could be insufficient, leaving behind some bonding. Sfrondini et al. reported that the amount of bonding remaining after lingual multibracket appliance therapy debonding is greater than that remaining after buccal multibracket appliance therapy [12]. Moreover, the inherent characteristics of the bonding agent used could influence the remaining bond [13]. Previously, the analysis of the enamel damage after multibracket appliance therapy debonding wash has been performed using scanning electron microscopy [14], profilometry [15], an atomic force microscope [16], and optic coherence tomography technology [17]. However, these methods are not possible to apply in clinical settings because they require the teeth to be extracted [14]. Therefore, non-invasive digital measurement procedures through intraoral scanning devices have been recommended to analyze the enamel damage accurately without needing to extract the teeth [18].

The aim of this study was to carefully explain the digital steps needed to quantify how much cement remains on the palatal tooth surface after debonding orthodontic therapy, how much enamel is removed when the cleaning procedure of the cement is carried out, and how much cement is left in the palatal surface of the tooth without being eliminated.

\section{Materials and Methods}

\subsection{Study Design}

The study was performed using 13 teeth from different dental sectors of the upper maxilla. The teeth did not present caries, restorations, prosthetic crowns, or fractures. Furthermore, they were extracted for orthodontic or periodontic reasons. The selection of the sample was performed at Alfonso X El Sabio University (Madrid, Spain), Alfonso $X$ El Sabio University (Madrid, Spain), and the Clinical Master Degree in Orthodontics at University of Salamanca (Salamanca, Spain) between September and November 2020. A randomized controlled trial was conducted based on the German Ethics Committee's statement for the use of organic tissues in medical research (Zentrale Ethikkommission, 2003) and was authorized by the Ethical Committee of the Faculty of Health Sciences, University Alfonso X el Sabio (Madrid, Spain) Faculty of Health Sciences, University Alfonso X el Sabio (Madrid, Spain) in July 2020 (Process No.08/2020). After giving informed consent, the patients agreed to transfer their teeth.

\subsection{Experimental Procedure}

The selected teeth were embedded into an epoxy resin model (Ref.: 20-8130-128. EpoxiCure ${ }^{\circledR}$, Buehler, IL, USA), following the manufacturer's recommendations, to simulate the dental arch. Afterward, the experimental model was scanned with an intraoral 
scan (True Definition, 3M ESPE TM, Saint Paul, MN, USA) to obtain a standard tessellation language (STL) digital file (STL1) (Figure 1a). Then, the fixed lingual multibracket appliance therapy was bonded to the palatal enamel surface via a single operator using a three-step adhesive technique. An acid etched agent (Ortho Solo ${ }^{\mathrm{TM}}$, Ormco Corporation, CA, USA) was applied to the palatal enamel surface for $20 \mathrm{~s}$. Afterward, the acid etching (Ortho Solo ${ }^{\mathrm{TM}}$, Ormco Corporation, CA, USA) was removed under profuse washing and dried. Furthermore, a photo-polymerized resin adhesive primer (Unitek Transbond ${ }^{\mathrm{TM}} \mathrm{XT}$, 3M ESPE TM, Saint Paul, MN, USA) was gently applied using a microbrush (Plus slim, Microbrush International, Grafton, MA, USA) on the previously etched palatal enamel surface and photopolymerized (BluePhase G2 TM, Ivoclar Vivadent, Schaan, Principado de Liechtenstein). The fixed lingual multibracket therapy was directly cemented from the second premolar to the second premolar following the instructions of the fabricant using a photo-polymerized composite resin cement (Transbond ${ }^{\mathrm{TM}}$ XT, 3M ESPE TM, Saint Paul, MN, USA). After that, the experimental model was submitted to a digital impression through an intraoral scan (True Definition, 3M ESPE TM, Saint Paul, MN, USA) (STL2) (Figure 1b). Next, the fixed lingual multibracket appliance was de-bonded using bracket-remover Weingart pliers (Carl Martin, Solingen, Germany), and a digital impression (True Definition, 3M ESPE TM $^{\mathrm{T}}$, Saint Paul, MN, USA) was made, obtaining the STL3 (Figure 1c). Afterward, the remaining cement of the fixed lingual multibracket appliance was removed from the palatal surfaces of the teeth using a composite bur (H379AGK, KOMET Dental, Lemgo, Germany) mounted on a micro-motor handpiece (INTRA MULTIflex L181M, KaVo, Biberach an der Riss, Germany) with profuse irrigation until no cement remnants were visible to the clinician's eye. Finally, a digital impression (True Definition, 3M ESPE TM, Saint Paul, MN, USA) of the experimental model was produced (STL4) (Figure 1d).

a)
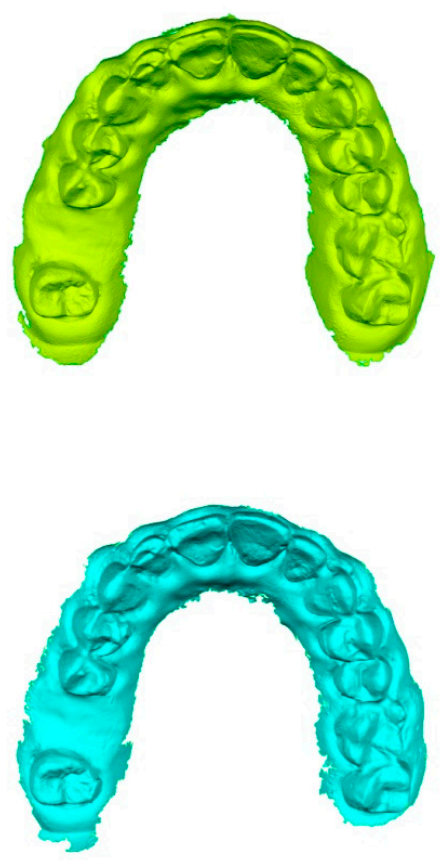

c)

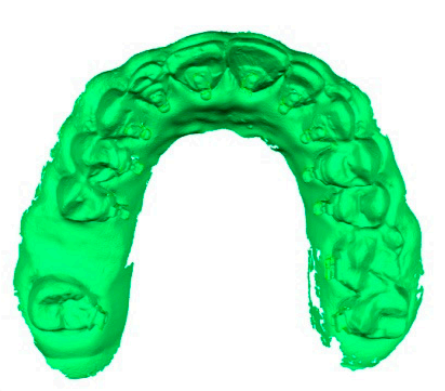

b)

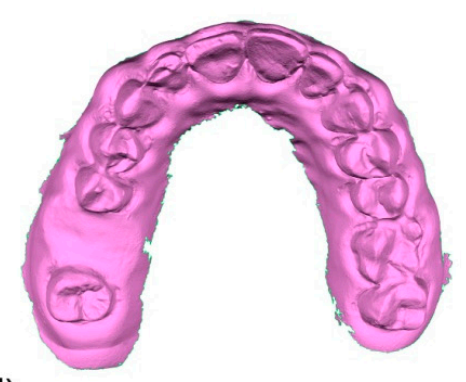

d)

Figure 1. Occlusal perspective of the STL1 (a), STL2 (b), STL3 (c), and STL4 (d) digital files.

\subsection{Alignment Procedure}

Once all the standard tessellation language (STL) digital files were obtained (STL1-4), they were exported into the engineering software Geomagic Wrap (3D Geomagic Capture Wrap, 3D Systems, Rock Hill, SC, USA), which allowed us to create and analyze the 3D images. Then, the STL digital files from 1 to 4 were aligned, using the non-modified buccal and oclusal surfaces as a reference to ensure an alignment procedure was used for the 
digital files. Later, all STL digital files of the teeth from 1.5 to 2.5 were digitally segmented to minimize the error rate in the alignment procedure (Figure $2 \mathrm{a}-\mathrm{d}$ ).

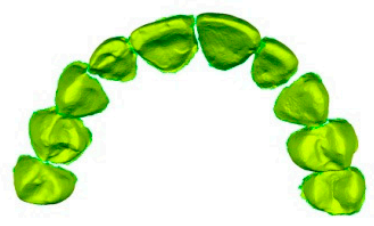

a)

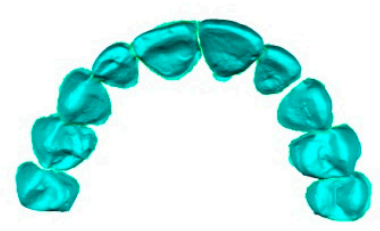

c)

Figure 2. Occlusal view of the individually segmented teeth from 1.5 to 2.5 of the standard tessellation language (STL)1 (a), STL2 (b), STL3 (c), and STL4 (d).

In addition, the fixed lingual multibracket appliances were scanned via micro Computed Tomography scan (micro-CT) (Skyscan 1176, Bruker-MicroCT, Kontich, Belgium) (STL5) with the following exposure parameters-160.0 kilovolt peak, 56.0-58.0 microamperes, $500.0 \mathrm{msec}$, 720 projections, 4 frames, a tungsten target between 0.25 and $0.375 \mathrm{~mm}$, a 3- $\mu \mathrm{m}$ resolution, and a pixel size of $0.127 \mu \mathrm{m}$ - to allow an accurate boundary in the STL digital file alignment based on the cement remaining after the debonding procedure (Figure 3a-e).

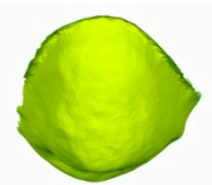

a)

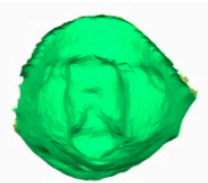

b)

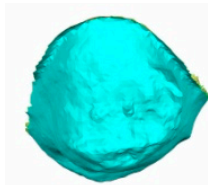

c)

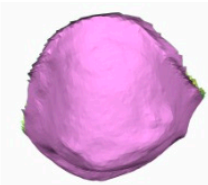

d)

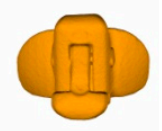

e)

Figure 3. Palatal view of STL1 (a), STL2 (b), STL3 (c), and STL4 (d) digital files from segmented tooth 1.5 and STL5 (e) from the corresponding fixed lingual multibracket appliances.

After segmenting the STL digital files (STL1-4), the files were re-aligned individually because the previously aligned but non-segmented STL digital files showed a low alignment rate (Figure $4 \mathrm{a}-\mathrm{c}$ ). However, the segmented and re-aligned STL digital files (STL1-4) showed a higher alignment rate, which could influence the accuracy of the measurement procedure for the cement remaining after lingual multibracket therapy debonding, the amount of orthodontic cement necessary to fix the lingual multibracket appliances, and the amount of enamel removed after lingual multibracket therapy debonding (Figure $4 \mathrm{~d}-\mathrm{f}$ ). The spectrum was set to $\pm 100 \mu \mathrm{m}$ and the tolerance was set to $\pm 10 \mu \mathrm{m}$ (Figure $4 \mathrm{~g}$ ). 


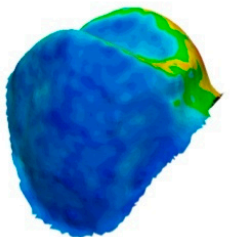

a)

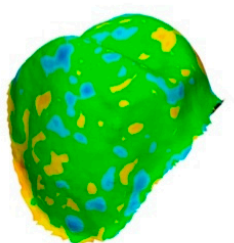

d)

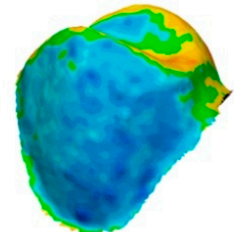

b)

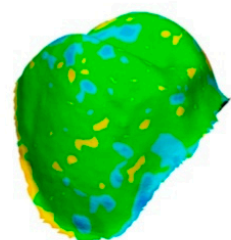

e)

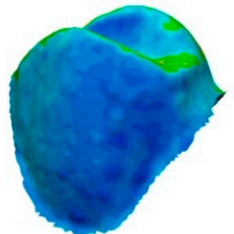

c)

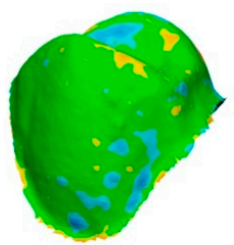

f)

Figure 4. Buccal perspective of the alignment rate of the segmented and aligned STL1 and STL2 (a), STL1 and STL3 (b), and STL1 and STL4, and (d) the buccal view of the segmented and re-aligned (a) STL1 and STL2 (d), STL1 and STL3 (e), and STL1 and STL4 (f). Reddish colors indicate an increase in volume, bluish colors indicate a decrease in volume, and green represents an accurate alignment (g).

\subsection{Measurement Procedure}

All measurements were obtained from the tooth 1.5 after the randomization procedure (Epidat 4.2, Galicia, Spain). Moreover, the STL5 digital file from the micro-CT (Skyscan 1176, Bruker-MicroCT, Kontich, Belgium) scan was positioned on tooth 1.5 to obtain a more accurate 3D image. The measurement procedure for the volume and area of cement remaining after lingual multibracket therapy debonding was performed by comparing the STL1 (Figure 5a) and STL3 (Figure 5b) digital files. A Boolean operation was used to achieve a boundary in the previously segmented STL1 (Figure 5c) and STL3 (Figure 5d). To identify the boundaries between both STL digital files, the STL3 was slightly over-contoured, the Boolean operation with the subsequent boundary was obtained, and then the STL3 was slightly under-contoured to its original size. Then, the area of cement remaining in the STL1 digital file was selected (Figure 5e), and a new polygon of the selected area was obtained (Figure 5g). Moreover, the area of cement remaining was selected in the STL3 through boundary selection (Figure 5f), and a new polygon was obtained (Figure 5h). The normals of this polygon were flipped, and a new polygon was created by merging the areas of Figure $5 \mathrm{~g}$, h, thereby obtaining a closed volume that corresponds to the remaining cement after fixed lingual multibracket appliance debonding (Figure 5i).

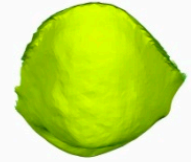

a)

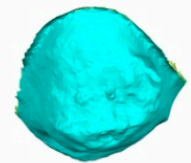

b)

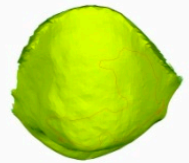

c)

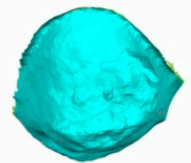

d)

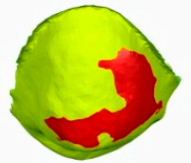

e)

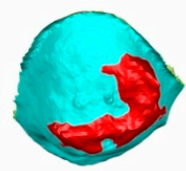

f)

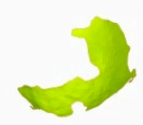

g)

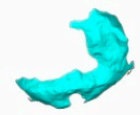

i)

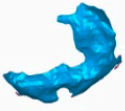

h)

Figure 5. Palatal perspective of tooth 1.5 segmented on STL1 (a) and STL3. (b) Boundary creation on STL1 (c) and STL3. (d) Boundary selection of the cement remaining on STL1 (e) and STL3. (f) Mesh of the cement remaining on STL1 (g) and STL3 (h), and the combined meshes with the remaining cement after bracket removal from STL1 and STL3, obtaining a closed polygon (i). 
The volume of the cement remaining in the STL3 digital file (Figure 6a) and the mesh of the cement remaining in the STL4 digital file after cement removal (Figure 6b) for the previously segmented and re-aligned tooth 1.5 were selected and compared to analyze the volume and area of the excess cement that was not removed after cement clearance and left in the palatal area of the tooth, by means of an intersection Boolean operation (3D Geomagic Capture Wrap, 3D Systems@ $\odot$, Rock Hill) (Figure 6c).

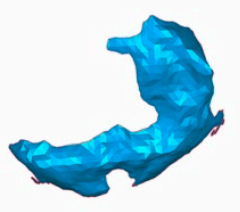

a)
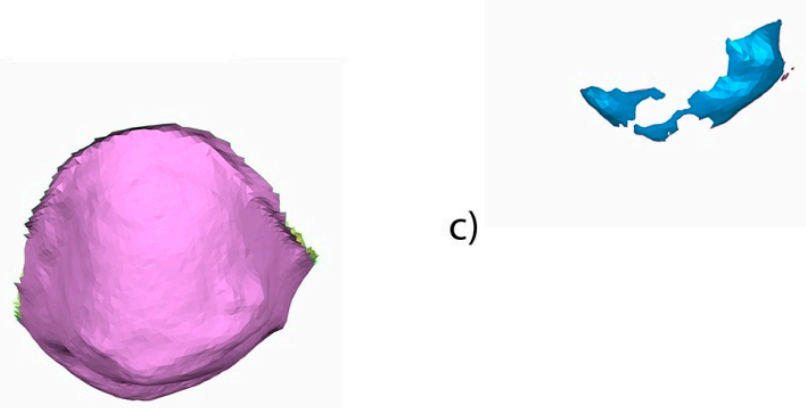

c)

b)

Figure 6. Mesh of the remaining cement after fixed lingual multibracket appliance debonding present in STL3 (a), palatal perspective of STL4 (b), and mesh of the remaining cement after cement removal in STL4 (c).

Moreover, these measurement procedures were also used to analyze the volume and area of enamel damage after cement clearance by comparing the STL1 and STL4 digital files (Figure 7a-i).

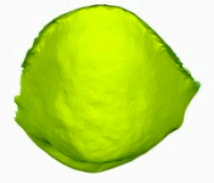

a)

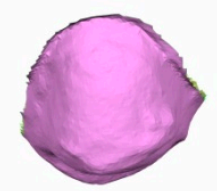

b)

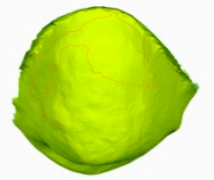

c)

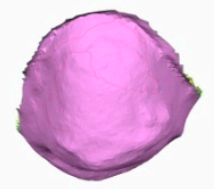

d)

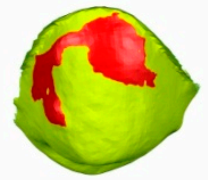

e)

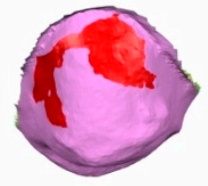

f)

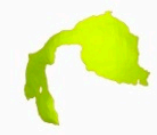

g)

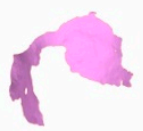

i)

h)

Figure 7. Palatal perspective of tooth 1.5 segmented on STL1 (a) and STL4. (b) Slight over-contouring of STL1 (c) and STL4. (d) Boundary of the enamel removed from STL1 (e) and STL4. (f) Mesh of the enamel removed from STL1 (g) and STL4 (h) and the combined meshes with the enamel removed after polishing STL1 and STL4, obtaining a closed polygon (i).

Additionally, the volume of orthodontic cement used to fix the lingual multibracket appliances was also measured by re-aligning the STL1 and STL2 of the previously segmented tooth 1.5, as well as the STL5 digital file from the micro-CT (Skyscan 1176, Bruker-MicroCT, Kontich) scan. The STL2 digital file (Figure 8a) was digitally filled to obtain a solid polygon 
(Figure $8 \mathrm{~b}$ ), and the bracket cemented in the STL2 digital file was digitally removed using a subtractive Boolean operation with the corresponding bracket of the STL5 digital file (Figure 8c). Then, a solid polygon was obtained from the previously segmented and realigned tooth 1.5 with the volume of the orthodontic cement used to fix the lingual bracket appliance (Figure 8c).

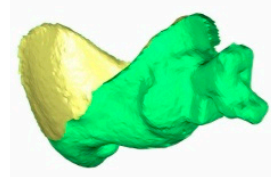

a)

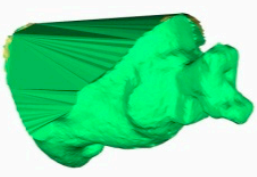

b)

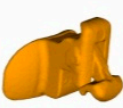

c)

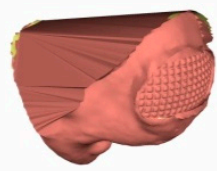

d)

Figure 8. Proximal perspective of tooth 1.5 segmented on STL2 (a) and the solid mesh of tooth 1.5 on STL2. (b) STL5 (c) and the solid mesh of tooth 1.5 on STL2 with the volume of the cement used to adhere and an absent bracket $(\mathbf{d})$.

Finally, the STL1 digital file (Figure 9a) and the new digital mesh of the previously segmented and re-aligned tooth 1.5 with the volume of the orthodontic cement used to fix the corresponding lingual bracket appliance (Figure 9b) were selected to analyze the volume of the orthodontic cement used to fix the corresponding lingual bracket appliance. The measurement procedure (Figure 9c-i) was performed with the same protocol used in the previous steps by employing the volume and area of cement remaining after lingual multibracket therapy debonding.
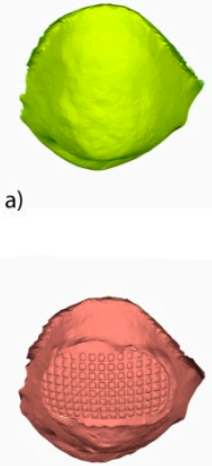

b)

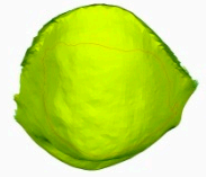

c)

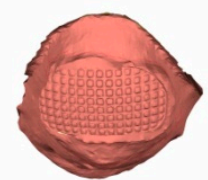

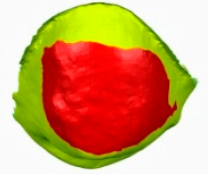

e)

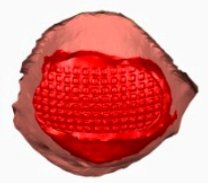

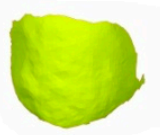

g)

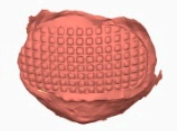

i)

Figure 9. Palatal perspective of the tooth 1.5 segmented on STL1 (a), solid mesh of tooth 1.5 on STL2 with the volume of the cement used to adhere and the absence of a bracket. (b) Slight over-contouring of STL1 (c) and the mesh of STL2 without a bracket. (d) Boundary of the orthodontic cement used to fix the corresponding lingual bracket appliance of STL1 (e) and the mesh of STL2 without a bracket. (f) Mesh of the orthodontic cement used to fix the corresponding lingual bracket appliance on STL1 (g) and the mesh of STL2 (h) and combined meshes with the orthodontic cement used to fix the corresponding lingual bracket appliance, obtaining a closed polygon (i).

\subsection{Validation of the Repeatability and Reproducibility}

To validate the repeatability of this new digital technique, the volume and area of the cement remaining after lingual multibracket therapy debonding, the volume of the cement remaining after cement clearance, and the volume of the enamel removed after cement clearance were calculated two times by the same operator (Operator A). In addition, the area and volume measurements were performed two times by a different operator (Operator B) to validate the reproducibility of the morphometric measurement technique. 


\subsection{Statistical Tests}

The variables of interest were registered for statistical analysis (SPSS 22.00, Microsoft inc, Redmond, WA, USA). Descriptive statistics were expressed as the mean and standard deviation (SD) values for quantitative variables. Comparative statistics were employed by comparing the intra-operator and inter-operator measurements using an ANOVA test. The statistical significance was set at $p<0.05$. In addition, a Gage $R \& R$ statistical analysis was done to analyze the repeatability and reproducibility of the morphometric measurement technique.

\section{Results}

The means and standard deviation (SD) values for the area of the cement remaining after lingual multibracket therapy debonding, the area of the cement remaining after cement clearance, and the area of the enamel removed after cement clearance are displayed in Table 1.

Table 1. Descriptive statistics of the area of the cement remaining after lingual multibracket therapy debonding, the area of the cement remaining after cement clearance, and the area of the enamel removed after cement clearance.

\begin{tabular}{ccccccc}
\hline Operator & I & $\boldsymbol{n}$ & Mean & SD & Minimum & Maximum \\
\hline \multirow{2}{*}{ A } & 1 & 3 & 8.769 & 3.221 & 5.876 & 12.240 \\
& 2 & 3 & 8.631 & 3.146 & 5.862 & 12.052 \\
B & 1 & 3 & 8.653 & 3.222 & 5.769 & 12.131 \\
& 2 & 3 & 8.679 & 3.111 & 5.749 & 11.945 \\
\hline
\end{tabular}

SD: standard deviation.

The ANOVA statistical analysis of the morphometric measurement technique after segmenting and re-aligning the STL digital files based on the area of the cement remaining after lingual multibracket therapy debonding, the area of the cement remaining after cement clearance, and the area of the enamel removed after cement clearance revealed no statistically significant differences between the two measurements performed by each operator $(p=0.601)$ or between the measurements performed between the two operators $(p=0.612)$.

The Gage R\&R statistical analysis of the morphometric measurement technique after segmenting and re-aligning the STL digital files based on the area of the enamel removed after cement clearance showed a repeatability value of $0.07 \%$. Repeatability values below $1 \%$ are necessary to demonstrate high repeatability. Therefore, the morphometric measurement technique demonstrated a high repeatability rate for the area measurement (Figures 10 and 11). In addition, the Gage R\&R statistical analysis of the morphometric measurement technique after segmenting and re-aligning the STL digital files based on the area of the cement remaining after lingual multibracket therapy debonding, the area of the cement remaining after cement clearance, and the area of the enamel removed after cement clearance performed by the two operators showed a reproducibility value of $0.00 \%$. Reproducibility values below $1 \%$ are necessary to demonstrate high reproducibility. Therefore, the morphometric measurement technique demonstrated a high reproducibility rate for the area measurement (Figures 10 and 11).

The means and SD values for the volume of the cement remaining after lingual multibracket therapy debonding, the area of the cement remaining after cement clearance, and the area of the enamel removed after cement clearance are displayed in Table 2.

The ANOVA statistical analysis of the morphometric measurement technique after segmenting and re-aligning the STL digital files in terms of the volume of the cement remaining after lingual multibracket therapy debonding, the volume of the cement remaining after cement clearance, and the volume of the enamel removed after cement clearance revealed no statistically significant differences between the two measurements performed 
by each operator $(p=0.413)$ or between the measurements performed between the two operators $(p=0.697)$.

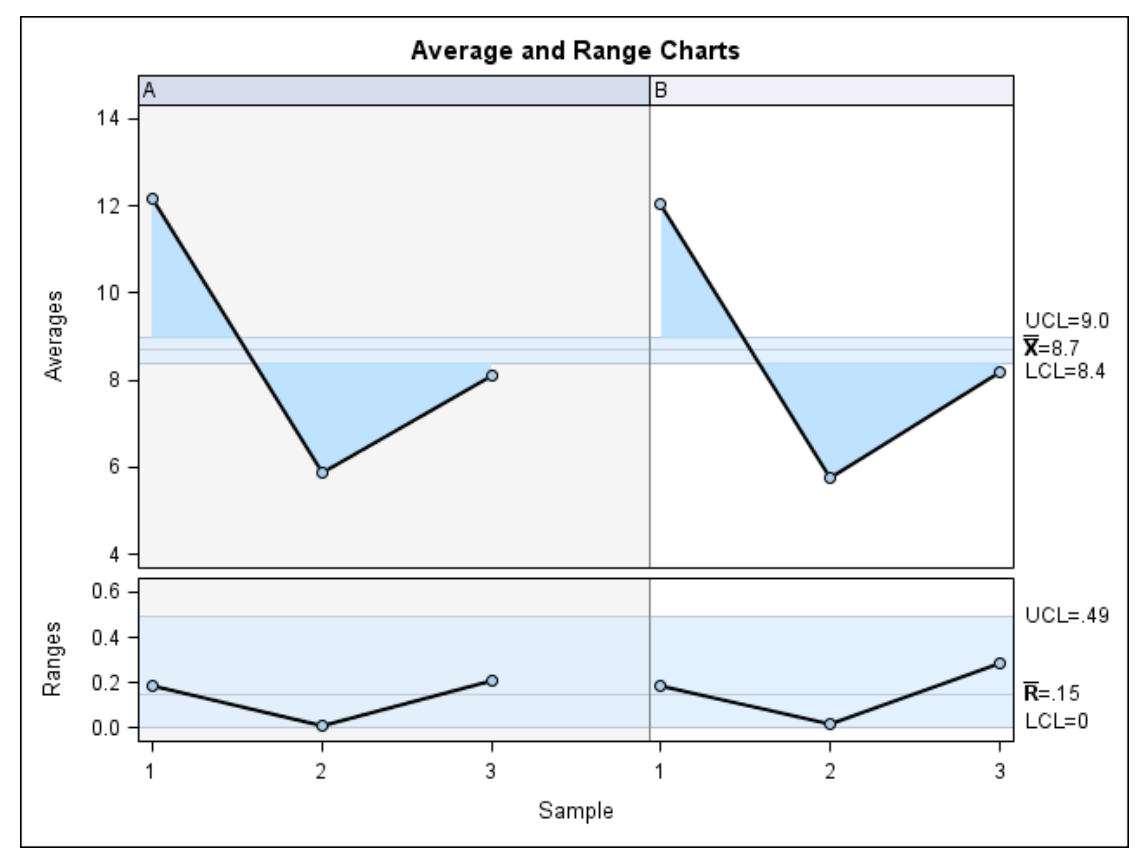

Figure 10. Charts of the average of two measurements of the cement area remaining after lingual multibracket therapy debonding (1), the cement area remaining after cement clearance (2), and the enamel area removed after cement clearance (3) performed by each operator (average) and the differences of the two measurements of the three area parameters determined by each operator (ranges).

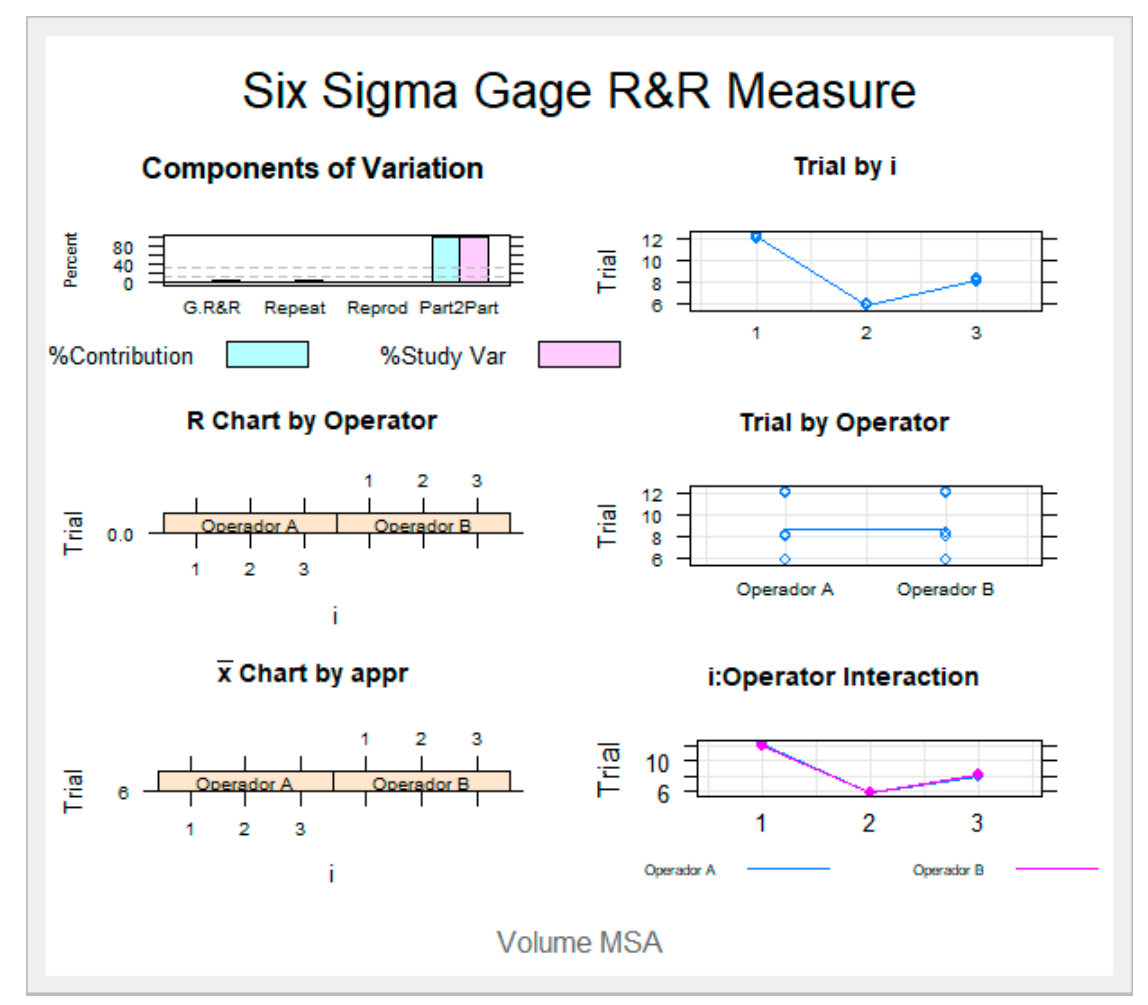

Figure 11. Measurement system analysis related to the area measurement with a chart of the contribution of each component to the total variance (Components of Variation), the mean control chart and the range control chart (R Chart by Operator and $x$ Chart by appr), every measurement point in the graph (Trial by I and Trial by Operator), and interactions between the operators (i): Operator interaction). 
Table 2. Descriptive statistics of the volume of the cement remaining after lingual multibracket therapy debonding, the volume of the cement remaining after cement clearance, and the volume of the enamel removed after cement clearance.

\begin{tabular}{ccccccc}
\hline Operator & I & $\boldsymbol{n}$ & Mean & SD & Minimum & Maximum \\
\hline \multirow{2}{*}{ A } & 1 & 3 & 0.986 & 0.708 & 0.428 & 1.783 \\
& 2 & 3 & 0.967 & 0.702 & 0.421 & 1.759 \\
B & 1 & 3 & 0.967 & 0.712 & 0.412 & 1.769 \\
& 2 & 3 & 0.975 & 0.680 & 0.423 & 1.735 \\
\hline
\end{tabular}

SD: standard deviation.

The Gage R\&R statistical analysis of the morphometric measurement technique after segmenting and re-aligning the STL digital files based on the volume of the cement remaining after fixed lingual multibracket therapy debonding, the volume of the cement remaining after cement clearance, and the volume of the enamel removed after cement clearance showed a repeatability value of $0.16 \%$. Repeatability values below $1 \%$ are necessary to demonstrate high repeatability. Therefore, the morphometric measurement technique demonstrated a high repeatability rate for the volume measurement (Figures 12 and 13). In addition, the Gage R\&R statistical analysis of the morphometric measurement technique after segmenting and re-aligning the STL digital files based on the volume of the cement remaining after fixed lingual multibracket therapy debonding, the volume of the cement remaining after cement clearance, and the volume of the enamel damage after cement clearance, as determined by the two operators, presented a reproducibility value of $0.00 \%$. Values below $1 \%$ are necessary to demonstrate high reproducibility. Therefore, the morphometric measurement technique shows a high reproducibility rate for the area measurement (Figures 12 and 13).

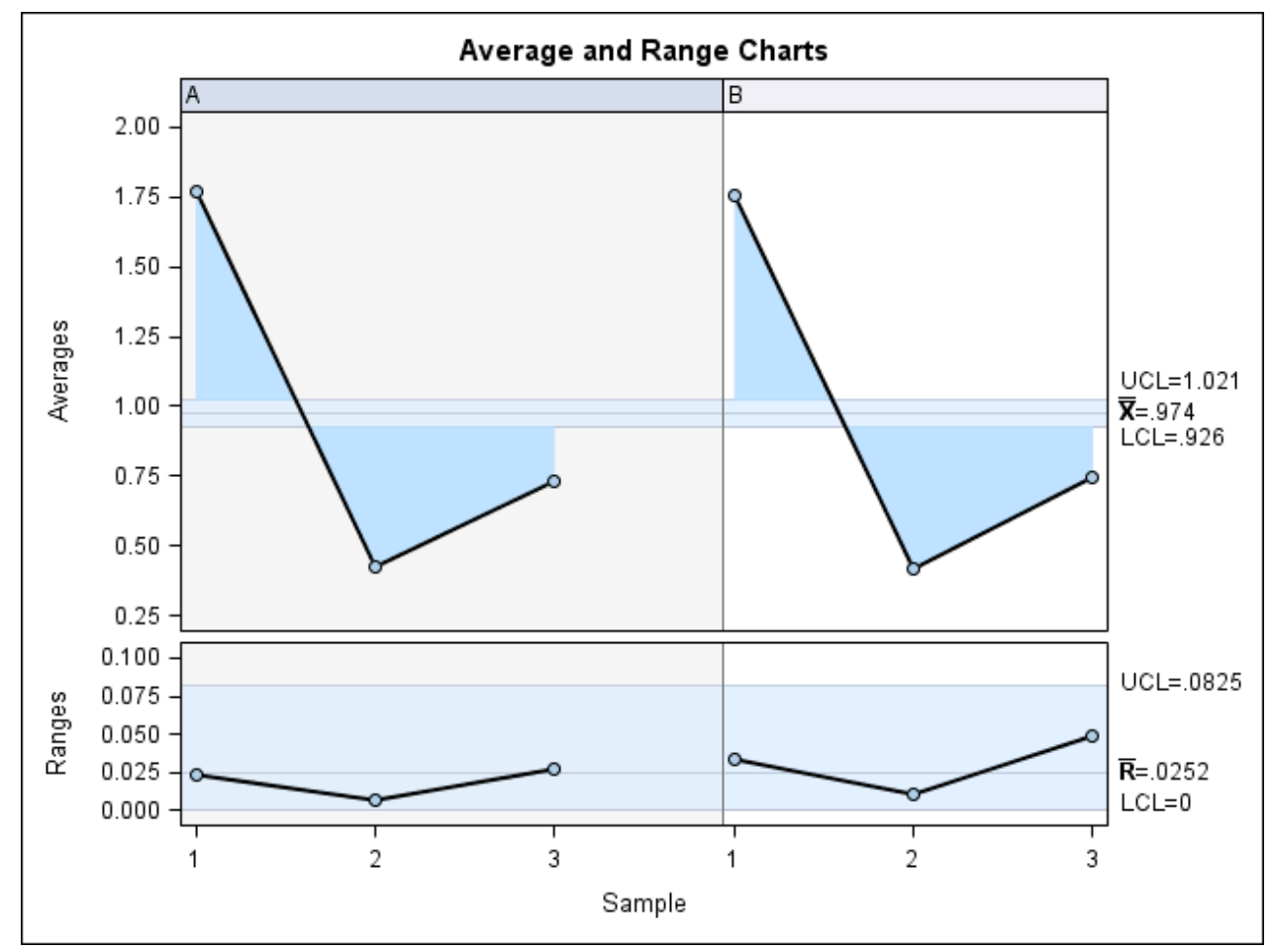

Figure 12. Charts for the average of the two measurements of the cement volume remaining after fixed lingual multibracket therapy debonding (1), the cement volume remaining after clearing the excess cement (2), and the enamel volume removed after cement clearance, (3) performed by each operator (average), as well as the differences in the two measurements of the three volume parameters determined by each operator (ranges). 


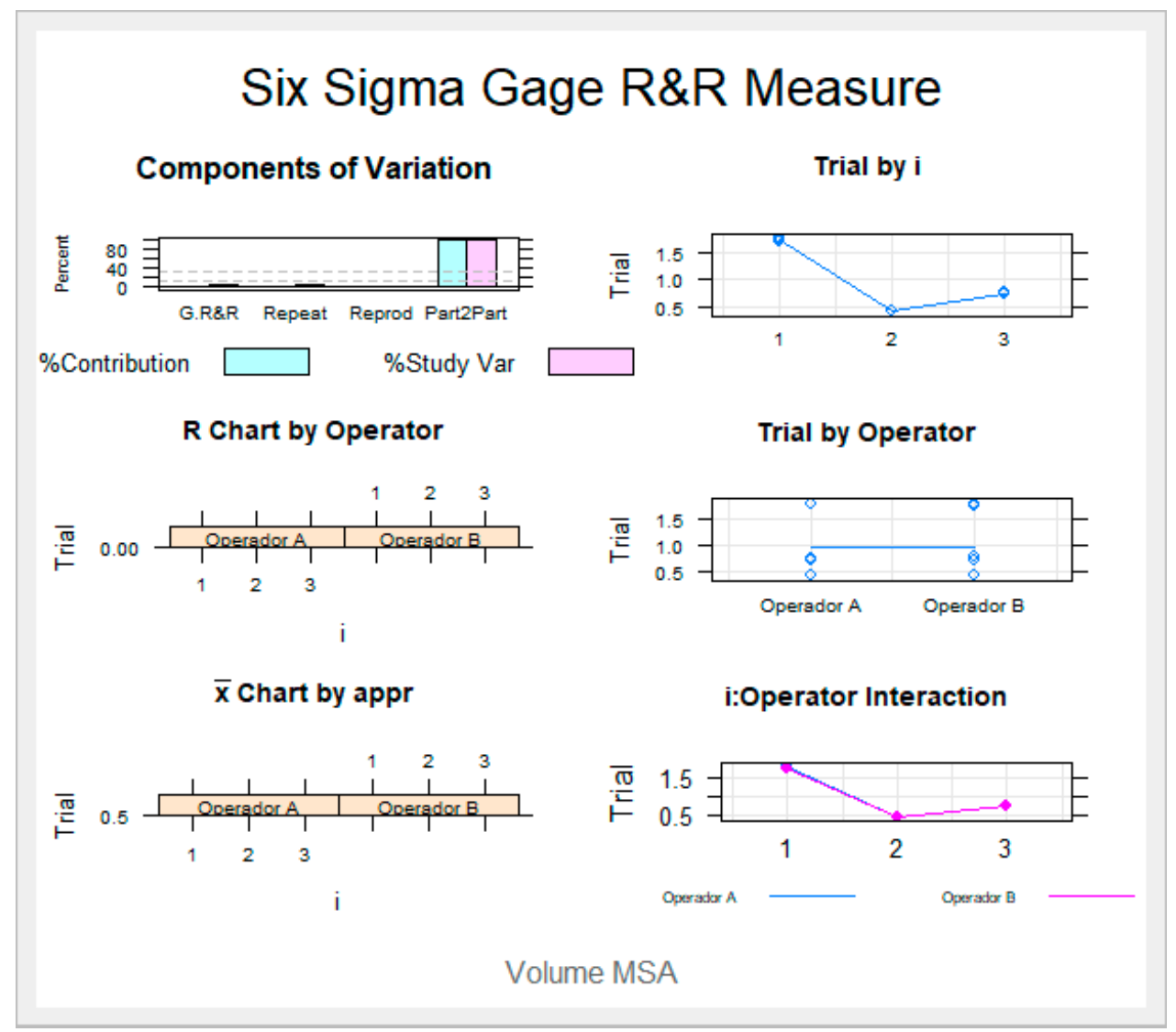

Figure 13. Measurement system analysis related to the volume measurement with a chart of the contribution of each component to the total variance (Components of Variation), a mean control chart and a range control chart ( $\mathrm{R}$ Chart by Operator and $x$ Chart by appr), every measurement point in the graph (Trial by I and Trial by Operator), and the interactions between the operators (i): Operator interaction).

\section{Discussion}

Enamel damage has been widely analyzed using clinical indexes, such as the enamel damage index (EDI), line angle grooves (LAG) [19], and the surface enamel index (ESI) [20]. Cement remains can also be evaluated by different scores of the index based on visual observations-using the adhesive remnant index (ARI) [15] and the composite residual (CR) [21]. The ARI has been evaluated by digital photography and disclosing tablets, showing an absence of reproducibility among operators [22]. In addition, the remnant index was previously used with optical microscopy techniques to evaluate the cement remaining after the debonding of lingual customized brackets [23]. Even though magnification showed less enamel damage and fewer cement remains after debonding [14], the use of a visual index to assess these nanometric volumes is subject to the opinion of the operator [18] Light sources have also been used to visually analyze cement remains, specifically using conventional or fluorescence light [24], which depends on the fluorescent ability of the orthodontic cement to identify resin remnants under direct view [17]. However, light source techniques have shown no statistically significant differences in cement removal detection [20]. Spectrophotometric measurement techniques have also been used to evaluate enamel damage after fixed multibracket appliance therapy and are associated with the resin tag depth confirmed in the bonding of brackets [25]. However, none of the methods previously described can accurately measure the area and volume of the cement remaining and enamel removed after fixed lingual multibracket appliance therapy debonding.

Presently, technological advances allow one to analyze nanometric surfaces and quantify nanometric volumes that are imperceptible to the clinician's eye. Scanning electron microscope (SEM) technology has been used to analyze the enamel roughness after fixed 
lingual multibracket appliance therapy debonding by comparing different polishing techniques ([16]) and orthodontic cements [26]. This measurement technique has also allowed the evaluation of dental fractures in the dental enamel, as well as the presence of ledges or scratch lines [27]. However, the measurement technique is based on the analysis of pixels in a previously obtained 2D image [28]. Therefore, the SEM technique does not allow one to quantitatively measure volumes [21] Hence, other measurement techniques are required to accurately analyze the volumes of cement remaining and the enamel removed after fixed lingual multibracket appliance therapy debonding. Chan et al. reported a measuring procedure using optical coherence tomography (OCT) to analyze the depth of scanned volumes [29]. Moreover, Leäo et al. used OCT technology to compare 3D images from multibracket appliance therapy debonding to measure the volume and remaining areas [17]. However, some other measurement techniques have also been used, such as atomic force microscopy to perform a nanometric quantification of enamel surface roughness [30], planner sulphometry [31], and profilometry [32] to measure the enamel loss. In the present study, the morphometric measurement technique was used to accurately measure nanometric volumes and areas. This technique is the only one demonstrated to be reproducible and repeatable, allowing one to accurately measure the nanometric areas and volumes of the cement remaining and enamel removed after fixed lingual multibracket appliance therapy debonding by quantifying the normal factors of the Tessella network of the cement remaining and the enamel removed between the STL digital files after the re-aligning procedure.

In addition, Renne et al. reported that the digital scanner and digital impression technique used could influence the accuracy of the measurement procedure. For this purpose, the 3Shape D800 digital scanner showed the highest truth values in full arch digital impressions, followed by the CEREC Bluecam digital scanner, while the Carestream 3500 digital scanner showed the highest accuracy results in full arch digital impressions, which was followed by the CEREC Blueacam digital scanner. However, the best compromise between working time and accuracy was provided with the 3Shape TRIOS digital scanner [33]. Treesh et al. reported the Carestream CS 3500 digital scanner to have the lowest accuracy and truth results in full arch digital impressions and also reported a significantly higher working time compared to the CEREC Omnicam digital scanner and TRIOS color digital scanner [34]. In addition, Ender et al. analyzed the accuracy of eight intraoral digital scanners, comparing partial-arch and full-arch digital impressions by aligning the STL digital files and measuring the differences between the normal factors of the Tessella network. The authors reported statistically significant differences $(p<0.05)$ between the truth values of the partial-arch and total-arch digital impressions with the total-arch digital impressions found to be less accurate than the partial-arch digital impressions [35]. Therefore, in the present study, the teeth were individually segmented and re-aligned. Moreover, Zimmermann et al. reported that digital impressions are more accurate than conventional alginate impressions, suggesting a promising future for digital impressions in orthodontics [36]. It was also noted that the accuracy of the STL digital files obtained from intraoral scanners is significantly better $(p<0.05)$ in powder-dependent scanners than non-powder-dependent scanners because translucency produces few errors in the images [37]. Therefore, in the present study, we used a power-dependent intraoral scanner.

Several factors can affect the accuracy of a digital scanner, and these drawbacks can be carried over to the STL digital file, thereby increasing the cumulative error rate caused by each alignment procedure, making it essential to avoid measurement errors in segmenting and re-alignment procedures. Moreover, the density of the Tessella network provided by the digital scan device can influence the accuracy of the measurement procedure. Therefore, it is important to previously analyze the characteristics of the digital scan device before using the device to make a digital impression.

A fixed lingual multibracket appliance was selected due to the relevance of cement remaining on the lingual surfaces of teeth and the difficulties related to the bonding, debonding, and polishing procedures associated with lingual orthodontics. The chosen ad- 
hesion technique for fixed lingual multibracket appliances is considered very important in orthodontics. However, completely removing the remaining cement is still a challenge [38], especially when the visual detection of remaining cement is compromised, increasing the probability of cement remaining on the tooth and/or excessive enamel loss. In response, the morphometric technology was shown to be a repeatable and reproducible measurement technique to accurately analyze the capability to remove the remaining enamel after fixed multibracket appliance therapy debonding, thereby improving the techniques and materials actually employed to remove the remaining cement without damaging the enamel surface.

\section{Conclusions}

Within the limitations of this study, the results show that the morphometric measurement technique is a reproducible, repeatable, and accurate method for quantifying the volume and area of cement excess after debonding lingual fixed multibracket therapy, the volume and area of enamel removed during the clearance step of the excess cement, the volume and area of the cement that was not removed from the palatal surface of the tooth, and the volume of cement used to adhere the lingual fixed multibracket therapy.

Author Contributions: Conceptualization, C.G.-B.; methodology, A.B.M., A.A.L., and J.A.P.-B. software, J.A.P.-B.; validation, Á.Z.-M.; resources, A.C.; writing—review and editing, A.A.M.; visualization, Á.Z.-M.; supervision, J.A.P.-B.; project administration, Á.Z.-M. All authors have read and agreed to the published version of the manuscript.

Funding: This research received no external funding.

Institutional Review Board Statement: Not applicable.

Informed Consent Statement: Not applicable.

Data Availability Statement: Data available on request due to restrictions eg privacy or ethical.

Conflicts of Interest: The authors declare no conflict of interest.

\section{References}

1. Beyling, F.; Schwestka-Polly, R.; Wiechmann, D. Lingual orthodontics for children and adolescents: Improvement of the indirect bonding protocol. Head Face Med. 2013, 9, 27. [CrossRef] [PubMed]

2. Hohoff, A.; Wiechmann, D.; Fillion, D.; Stamm, T.; Lippold, C.; Ehmer, U. Evaluation of the parameters underlying the decision by adult patients to opt for lingual therapy: An international comparison. J. Orofac. Orthop. 2003, 64, 135-144. [CrossRef] [PubMed]

3. Knösel, M.; VogelAlvarez, R.; Blanck-Lubarsch, M.; Helms, H.J. Comparison of potential long-term costs for preventive dentistry treatment of post-orthodontic labial versus lingual enamel cavitations and esthetically relevant white-spot lesions: A simulation study with different scenarios. Head Face Med. 2019, 15, 22. [CrossRef] [PubMed]

4. Botzenhart, U.U.; Henningsen, A.; Quaas, S.; Luthardt, R.G.; Proff, P.; Spassov, A.; Gedrange, T. 3D assisted morphological analysis of lingual upper central and lateral incisor surfaces. Biomed. Tech. 2012, 57, 71-77. [CrossRef]

5. Mavrea, D.; Cuzin, J.F.; Boonen, G.; Vannet, B.V. The effect of various adhesives, enamel etching, and base treatment on the fail-ure frequency of customized lingual brackets: A randomized clinical trial. Eur. J. Orthod. 2018, 40, 249-253. [CrossRef]

6. Brosh, T.; Strouthou, S.; Sarne, O. Effects of buccal versus lingual surfaces, enamel conditioning procedures and storage dura-tion on brackets debonding characteristics. J. Dent. 2005, 33, 99-105. [CrossRef]

7. Lopes, M.S.; Pereira, D.L.; de Oliveira, C.C.B.; Magri, M.; Zezell, D.M.; Leonidas, A.S. The lingual enamel morphology and bracket shear bond strength influenced by Nd: YAG laser and aluminium oxide sandblasting preconditioning. Clin. Oral. Investig. 2020. [CrossRef]

8. Lombardo, L.; Kaplan, A.; Lapenta, R.; Bratti, E.; Pera, C.; Scuzzo, G.; Siciliani, G. A comparative study of lingual bracket bond strength. Orthodontics 2011, 12, 178-187.

9. Eichenberger, M.; Iliadi, A.; Koletsi, D.; Eliades, G.; Verna, C.; Eliades, T. Enamel Surface Roughness after Lingual Bracket Debond-ing: An In Vitro Study. Materials 2019, 12, 4196. [CrossRef]

10. Garg, R.; Dixit, P.; Khosla, T.; Gupta, P.; Kalra, H.; Kumar, P. Enamel Surface Roughness after Debonding: A Comparative Study using Three Different Burs. J. Contemp. Dent. Pract. 2018, 19, 521-526.

11. Janiszewska-Olszowska, J.; Tomkowski, R.; Tandecka, K.; Stepien, P.; Szatkiewicz, T.; Sporniak-Tutak, K.; Grocholewicz, K. Effect of orthodontic debonding and residual adhesive removal on 3D enamel microroughness. PeerJ. 2016, 4, e2558. [CrossRef] [PubMed] 
12. Sfondrini, M.F.; Gandini, P.; Gioiella, A.; Zhou, F.X.; Scribante, A. Orthodontic Metallic Lingual Brackets: The Dark Side of the Moon of Bond Failures? J. Funct. Biomater. 2017, 8, 27. [CrossRef] [PubMed]

13. David, V.A.; Staley, R.N.; Bigelow, H.F.; Jakobsen, J.R. Remnant amount and cleanup for 3 adhesives after debracketing. Am. J. Orthod. Dentofac. Orthop. 2002, 121, 291-296. [CrossRef] [PubMed]

14. Claudino, D.; Kuga, M.C.; Belizário, L.; Pereira, J.R. Enamel evaluation by scanning electron microscopy after debonding brackets and removal of adhesive remnants. J. Clin. Exp. Dent. 2018, 10, e248-e251. [CrossRef]

15. Faria-Junior, E.M.; Giraldo, R.D.; Berger, S.B.; Correr, A.B.; Correr-Sobrinho, L.; Ruiz Contreras, E.F.; Lopes, M.B. In-vivo evaluation of the surface roughnedd and morphology of enamel after bracket removal and polishing by different techniques. Am. J. Orthod. Dentofac. Orthop. 2015, 147, 324-329. [CrossRef]

16. Karan, S.; Kircelli, B.H.; Tasdelen, B. Enamel surface roughness after debonding: Comparison of two different burs. Angle Orthod. 2010, 80, 1081-1088. [CrossRef]

17. Leäo Filho, J.C.; Braz, A.K.; de Araujo, O.M.; Tanaka, O.M.; Pithon, M.M. Enamel quality after debonding: Evaluation by optical coherence tomography. Braz Dent. J. 2015, 26, 384-389. [CrossRef]

18. Zubizarreta-Macho, Á.; Triduo, M.; Alonso Pérez-Barquero, J.; Guinot Barona, C.; Albaladejo Martínez, A. Novel Digital Technique to Quantify the Area and Volume of Cement Remaining and Enamel Removed after Fixed Multibracket Appliance Therapy Debonding: An In Vitro Study. J. Clin. Med. 2020, 9, 1098. [CrossRef]

19. Baumann, D.F.; Brauchli, L.; van Waes, H. The influence of dental loupes on the quality of adhesive removal in orthodontic debonding. J. Orofac. Orthop. 2011, 72, 125-132. [CrossRef]

20. Cardoso, L.A.; Valdrighi, H.C.; Vedovello Filho, M.; Correr, A.B. Effect of adhesive remnant removal on enamel topography after bracket debonding. Dent. Press J. Orthod. 2014, 19, 105-112. [CrossRef]

21. Schott, T.C.; Meller, C. A new Fluorescence-aided Identification Technique (FIT) for optimal removal of resin-based bracket bonding remnants after orthodontic debracketing. Quintessence Int. 2018, 49, 809-813. [CrossRef] [PubMed]

22. Pont, H.B.; Özcan, M.; Bagis, B.; Ren, Y. Loss of surface enamel after bracket debonding: An in-vivo and ex-vivo evaluation. Am. J. Orthod. Dentofac. Orthop. 2010, 138, 387.e1-387.e9. [CrossRef]

23. Kuskonmaz, C.; De Stefani, A.; Artioli, G.; Zanarini, M.; Bonetti, G.A.; Bruno, G.; Gracco, A. The use of the laser confocal scanning microscopy to measure resin remnants on customized lingual bracket. BMC Oral Health 2020, 20, 142. [CrossRef] [PubMed]

24. Stadler, O.; Dettwiler, C.; Meller, C.; Dalstra, M.; Verna, C.; Connert, T. Evaluation of a Fluorescence-aided Identification Tech-nique (FIT) to assist clean-up after orthodontic bracket debonding. Angle Orthod. 2019, 89, 876-882. [CrossRef] [PubMed]

25. Zaher, A.R.; Abdalla, E.M.; Abdel Motie, M.A.; Rehman, N.A.; Kassem, H.; Athanasiou, A.E. Enamel colour changes after debonding using various bonding systems. J. Orthod. 2012, 39, 82-88. [CrossRef] [PubMed]

26. Alencar, E.Q.; Nobrega, M.L.; Dametto, F.R.; Santos, P.B.; Pinheiro, F.H. Comparison of two methods of visual magnification for re-moval of adhesive flash during bracket placement using two types of orthodontic bonding agents. Dent. Press J. Orthod. 2016, 21, 43-50. [CrossRef]

27. Rodríguez-Chávez, J.A.; Arenas-Alatorre, J.; Belio-Reyes, I.A. Comparative study of dental enamel loss after debonding braces by analytical scanning electron microscopy (SEM). Microsc. Res. Tech. 2017, 80, 680-686. [CrossRef]

28. Lombardo, L.; Martini, M.; Cervinara, F.; Spedicato, G.A.; Oliverio, T.; Siciliani, G. Comparative SEM analysis of nine F22 aligner cleaning strategies. Prog. Orthod. 2017, 18, 26. [CrossRef]

29. Chan, K.H.; Hirasuna, K.; Fried, D. Analysis of enamel surface damage after selective laser ablation of composite from tooth sur-faces. Photonics Lasers Med. 2014, 3, 37-45. [CrossRef]

30. ElSherifa, M.T.; Shamaa, M.S.; Montasser, M.A. Enamel around orthodontic brackets coated with flash-free and conventional adhe-sives. J. Orofac. Orthop. 2020, 81, 419-426. [CrossRef]

31. Hosein, I.; Sherriff, M.; Ireland, A.J. Enamel loss during bonding, debonding, and cleanup with use of a self-etching primer. Am. J. Orthod. Dentofacial. Orthop. 2004, 126, 717-724. [CrossRef] [PubMed]

32. Erdur, E.A.; Akın, M.; Cime, L.; İleri, Z. Evaluation of Enamel Surface Roughness after Various Finishing Techniques for Debonding of Orthodontic Brackets. Turk. J. Orthod. 2016, 29, 1-5. [CrossRef] [PubMed]

33. Renne, W.; Ludlow, M.; Fryml, J.; Schurch, Z.; Mennito, A.; Kessler, R.; Lauer, A. Evaluation of the accuracy of 7 digital scanners: An in vitro analysis based on 3-dimensional comparisons. J. Prosthet. Dent. 2017, 118, 36-42. [CrossRef]

34. Treesh, J.C.; Liacouras, P.C.; Taft, R.M.; Brooks, D.I.; Raiciulescu, S.; Ellert, D.O.; Grant, G.T.; Ye, L. Complete-arch accuracy of intraoral scanners. J. Prosthet. Dent. 2018, 120, 382-388. [CrossRef] [PubMed]

35. Ender, A.; Zimmermann, M.; Mehl, A. Accuracy of complete- and partial-arch impressions of actual intraoral scanning systems in vitro. Int. J. Comput. Dent. 2019, 22, 11-19. [PubMed]

36. Zimmermann, M.; Koller, C.; Rumetsch, M.; Ender, A.; Mehl, A. Precision of guided scanning procedures for full-arch digital impressions in vivo. J. Orofac. Orthop. 2017, 78, 466-471. [CrossRef]

37. Nedelcu, R.G.; Persson, A.S. Scanning accuracy and precision in 4 intraoral scanners: An in vitro comparison based on 3dimensional analysis. J. Prosthet. Dent. 2014, 112, 1461-1471. [CrossRef] [PubMed]

38. Mandall, N.A.; Hickman, J.; Macfarlane, T.V.; Mattick, R.C.; Millett, D.T.; Worthington, H.V. Adhesives for fixed orthodontic brackets. Cochrane Database Syst. Rev. 2018, 4, CD002282. [CrossRef] 